Voix et Images

\title{
Une mémoire déchirée, de Thérèse Renaud et Une voix pour Odile de France Théoret
}

\section{Madeleine Gagnon}

Volume 4, numéro 1, septembre 1978

Rina Lasnier

URI : https://id.erudit.org/iderudit/200143ar

DOI : https://doi.org/10.7202/200143ar

Aller au sommaire du numéro

Éditeur(s)

Les Presses de l'Université du Québec

ISSN

0318-9201 (imprimé)

1705-933X (numérique)

Découvrir la revue

Citer ce compte rendu

Gagnon, M. (1978). Compte rendu de [Une mémoire déchirée, de Thérèse

Renaud et Une voix pour Odile de France Théoret]. Voix et Images, 4(1), 143-146.

https://doi.org/10.7202/200143ar d'utilisation que vous pouvez consulter en ligne.

https://apropos.erudit.org/fr/usagers/politique-dutilisation/ 
Une mémoire déchirée ${ }^{1}$, de Thérèse Renaud et Une voix pour Odile ${ }^{2}$ de France Théoret

Une génération sépare ces deux récits qui s'offrent pourtant à la lecture la même année. Une même mémoire, déchirée. Mais non pas désespérée. La déchirure devient le creuset, l'antre, où l'écriture viendra recoudre les lambeaux d'une histoire écorchante - et quelle histoire ne l'a pas été - 
avec ce qui s'est forgé sur ces heurts, de lucidité analytique, d'intelligence poétique. L'écriture cicatrise. À la fois reconnaissance du lieu du manque, ces espaces incertains où déjà les premières paroles ne se tracent que d'un aveu à ne pas pouvoir reprendre l'événement - copier le réel - quel qu'en soit sa force; mais aussi réparation, puisque l'écriture c'est ce qui dans le sujet accepte de s'inscrire à côté, en déplacement continu; reliée mais parallèle à l'événement, pour en témoigner, mais encore parce que ce sillon perdu est le seul à signifier la fuite initiale des faits historiques dans la mémoire des petits, hommes et femmes.

La mémoire de ces deux textes est la même: prétexte à ce qui s'écrit, humblement conforme à ce qui la définit, une déchirure; le tissu événementiel ne diffère pas tellement non plus : par-delà la génération qui les sépare, il s'expose dans toute sa dureté et dit, à l'époque ici des grandes noirceurs d'avant la "révolution tranquille", ce qu'il en a été d'étouffant, d'opprimant, pour une petite fille, une adolescente, une femme, dont la conscience déjà pressentait l'ampleur de l'assujettissement.

Une même mémoire. Un même tissu événementiel. Pourtant, deux écritures radicalement différentes. Et c'est ici que se quittent les routes parties d'un même lieu. Peut-être à la fin se rejoindraient-elles en quelque endroit inexploré à cette heure par nos folles imaginations? Rien ne saurait le prévoir et rien, de toutes façons, n'oblige à le désirer.

Prenons d'abord la première route. Celle d'Une mémoire déchirée. Je dis d'abord, sans doute parce que le temps de l'événement précède l'autre, mais aussi, peut-être, parce que le choix de l'écriture nous est plus familier, plus connu, disons, plus traditionnel. Alors que dans les Sables du rêve ${ }^{3}$, Thérèse Renaud pratiquait déjà avec grande aisance et finesse l'écriture de rupture automatiste, celle d'ailleurs qui allait préparer la voie aux automatismes associatifs et réflexifs de l'écriture psychanalytique, ici elle choisit la voix de la conscience, laissant pulsions et fantasmes on ne sait trop à quelle dérive. Elle choisit la voix du témoignage direct, du je qui s'écrit sans les soubresauts de la fiction; la voix du cceur, comme disait André Brochu dans sa présentation du livre? Oui, la voix du cœur, mais si j'ajoutais que le cœur n'exclut ni la transposition poétique, ni la coupure du fantasme qui commande cette dernière, me pardonnerait-on? Cela dit, avec Une mémoire déchirée, Thérèse Renaud nous donne un témoignage historique, une espèce de document sociologique, d'une valeur inestimable. De cette époque, 1935-1955, nous ne connaissons trop souvent hélas que les restes calqués sur les idéologies dominantes: politiques, religieuses, littéraires. A part quelques écrits de rupture, autour du Refus global que signa d'ailleurs Thérèse Renaud, peu de voix firent entendre les effets aliénants des pouvoirs monolithes cléricaux sur le développement sexuel, affectif, intellectuel des enfants de ce temps. C'est ce que fait, avec vigueur et émotion, Thérèse Renaud. Je crois que tous les jeunes qui désirent 
savoir d'où nous venons, d'où ils viennent - et qui ne veut pas de ce savoir? - devraient plonger dans ce récit. Ils y découvriront la naissance d'une parole.

“J'écris d'où je viens. Je parle d'où je suis. Le passé ne m'intéresse que pour agiter l'avenir. L'ici et maintenant n'est pas aboli et n'est pas mémoire non plus, tout au plus une cage. Où es-tu ? ”

Ainsi débute Une voix pour Odile, qui, après Bloody Mary ${ }^{4}$, inscrit délibérément la fiction du je, c'est-à-dire, la conscience de la mémoire trouée qui agite (agit) le sujet historique dans la mise en cage de toute parole ultérieure. Mais cette page, cette mémoire blanchie par les ans, peut s'offrir à la quête poétique pour qu'en soient connus les effets d'interdits que constitue toute prise de parole. C'est ainsi que l'on dit de la lettre, dans sa livraison, qu'elle délivre. La lettre, l'ultime finalité de l'inconscient. Et l'écriture, des pulsions, des fantasmes, du rêve, s'en révèle son témoin privilégié.

Une voix pour Odile, c'est donc ce lieu dit de passage entre parole et écriture, passage que le jeu de la lettre permet. Inscrit dans une jeune tradition de recherches sur le mot (le formalisme) et la lettre (la psychanalyse), ce texte dit «à la lettre l'inexistence et le non-mouvement» (p. 66), cela qui fut barré au sujet qui s'éveille, "ressemblant à l'hystérie et au fascisme : la masochiste» (p. 67).

Écriture éminemment politique, une sorte de “plaidoyer pour le droit à l'existence des femmes», comme le propose le titre de la huitième partie. Un plaidoyer, oui, mais qui se méfie du nous militant ou propagandiste autre censure du sujet naissant - et qui a raison de s'en méfier. C'est simple, je n'écrit pas dans les marges des procès-verbaux d'un programme fictif d'une histoire utopiste. Je, si ça s'écrit, ne peut que renvoyer, inlassablement, aux strates historiques inscrites déjà dans l'assujettissement individuel. Je s'écrit parce que du très intime, ça peut dire calmement, par exemple: «Moi, France Théoret, je suis capable d'écrire depuis que mes mains m'apparaissent plissées" (p. 69); parce qu'aussi, peut-être, en ce procès abyssal, d'autres sujets pourront s'y reconnaître et se produire en écritures pour l'histoire des autres.

Une mémoire déchirée, la nôtre. Une voix pour Odile, pour toutes. $A$ tous. Tous ceux qui savent que l'écriture peut non seulement témoigner d'une réalité historique mais aussi transformer ses effets signifiants chez des sujets avides de changement.

Madeleine Gagnon.

1. Montréal, H.M.H., “L'Arbre „, 1978.

2. «Lecture en vélocipède/Les Herbes rouges », 1978. 
3. Publié d'abord par Les Cahiers de la file indienne, en 1946, et réédité dans les Herbes rouges, $n^{\circ} 29,1975$.

4. Les Herbes rouges, $n^{\circ} 45,1977$.

\section{Les Murs de Montréal de J.-P. Filion}

Avec les Murs de Montreal ${ }^{1}$ Jean-Paul Filion invite le lecteur à faire un voyage dans le temps - deuxième moment d'une odyssée qui remonte à l'enfance et dont Saint-André-Avellin, le premier cóté du monde avait livré les premières expériences.

Dès le titre on comprend que cet itinéraire, cet apprentissage est semé d'embùches, d'obstacles, d'écrans liés à Montréal qui dresse ses “murs" devant les aspirations et les ambitions du narrateur. Mais les images d'une ville ne sont jamais pure représentation. Elles expriment moins un lieu que la situation de l'individu face à ce lieu. Ainsi Montréal est tantôt « un paradis avec des fumées colorées qui se baraudent partout, des airs de moteurs par centaines et du monde comme [it n'a] jamais vu ça ». (p. 16) Tantôt "un champ de bataille à n'en plus finir" (p. 324). Vocabulaire affectif, voire hyperbolique qui souligne que la ville est toujours perçue subjectivement à travers les passions et les tensions qu'elle engendre.

Il ne faut donc pas s'attendre, en lisant ce récit de type biographique, a faire une "lecture s de Montréal. Certes, on y trouve surtout dans la première partie, à travers le regard d'un campagnard de quinze ans qui quitte son village pour venir travailler à la ville, l'approche globale de la grosse ville: ades maisons toutes collées, des automobiles toutes collées, des poteaux collés, le monde collé, assez qu'on ne pouvait pas voir le moindre petit trou nulle part * ( $p .17)$. Le regard se pose sur un espace encombré, rempli à l'excès et qui ne laisse à celui qui observe aucune échappée sur un espace non utilisé. Quelques lieux sont décrits : ceux du travail, ceux du logement et les rues où ils se trouvent. Cette vision met surtout l'accent sur les valeurs vitales ou affectives qu'implique la ville bien plus que sur les traits architecturaux ou esthétiques: enthousiasme, ennui, dégoût, désir de fuir un lieu où l'on étouffe, où l'on s'aliène : “ma journée finie, je rentre mort dans ma chambre de la rue Sherbrooke. Je vais de temps en temps au port voir les bateaux qui partent... Ou au mont Royal me guérir l'âme en me collant contre les arbres * (p. 54). Fuir, ce sera retourner à Saint-André-Avellin, dans le cocon familial mais les "murs" ne sont ni dans la ville, ni dans le village. Le narrateur apprendra qu'il les porte en lui comme des enveloppes que l'adolescent doit faire éclater pour trouver sa voie et se réconcilier avec lui-mème. 